\title{
Jnhmicro-texture of AZ31 Magnesium Alloy in Situ Tracking During Compressing Deformation
}

\author{
Zhongtang $\mathrm{WANG}^{1, \mathrm{a}}$, Zhijin $\mathrm{ZHAI}^{1, \mathrm{~b}}$ and Lingyi WANG ${ }^{2, \mathrm{c}}$ \\ ${ }^{1}$ School of Material Science \& Engineering, Shenyang Ligong University, Shenyang110159, China \\ ${ }^{2}$ Shenyang Institute of Technology, Liaoning Fushun113122, China \\ aztwang@imr.ac.cn', ,819155954@qq.com, ${ }^{\mathrm{c}}$ lywang01@sina.com
}

\begin{abstract}
Micro-texture evolvement mechanism of AZ31 magnesium alloy during compressing deformation had been studied in situ tracking method by $\operatorname{EBSD}$ (Electron backscatter diffraction). The micro-texture at same observation areas, which compressed three times continually at different deformation degree, had been investigated. The results presented that the micro-texture of AZ31 magnesium alloy rolling state is typical (0001) basic texture. When deformation degree was $11 \%$ and the temperature was $170^{\circ} \mathrm{C}$, grains orientation changed remarkably, and most grains had been twined fully and little grains twined partially. The initial basical rolling texture weakened gradually, and twined grains tropism accorded with $60 \%<10 \overline{1} 0>$ and $86.3 \%<\overline{1} 2 \overline{1} 0>$ orientation.
\end{abstract}

\section{Introduction}

Microstructure of Magnesium alloys is hep crystal structure, and it has a strong tendency of texture and anisotropy. Deformation mechanism of AZ31 Magnesium alloy is slip and twinning[1]. The cylindrical sliding and compression twin is main mechanism in the process of deformation[2-3]. AZ31 Magnesium alloy sheet, which rolled at temperature $300{ }^{\circ} \mathrm{C}$, presented large amounts of twin structure. Twins gradually disappeared and equiaxed grains appeared after annealing treatment[4]. Internal stress which occurred in twins related to surrounding stress field[5]. The main deformation mechanism of hot rolled AZ31B magnesium alloy sheet is basal slip, and it's easy to form single texture. The main deformation mechanism of cold rolled is the basal slip and extension twinning, and bimodal basal texture tilted to the rolling direction[6]. Asynchronous rolling can reduce the intensity of base texture of $\mathrm{Mg}$ alloy[7-8]. Grains misorientation angle which corresponded with base texture of AZ31 magnesium alloy is less than $30^{\circ}$, and grain misorientation distributed uniform and there was more small angle grain boundary, and grain size distributed uniform and was not easy to grow up[9]. The formability of the sheet will be improved by reducing the intensity of the base texture[10]. The twin types of magnesium during compression deformation included extension twin, and compression twin and secondary twin[11]. The mechanical property tests present that the activity of $\{1012\}<1011>$ twinning effected significantly on the yield stress and elongation rate in during compression[12]. Asynchronous rolling can decrease significantly the dynamic recrystallization temperature

* Corresponding author:ztwang@imr.ac.cn 
of AZ31 magnesium alloy, making grain refine and improve the low temperature rolling forming ability. Magnesium alloy sheet of $0.7 \mathrm{~mm}$ thickness had been manufactured without intermediate annealing. At the same time, the basal texture had been weakened by asynchronous rolling obviously, but the basal texture intensity does not monotonically decrease with the increasing of speed ratio[13]. The texture can be altered by changing deformation technology, and adding rare elements to weaken and randomize the texture of magnesium alloy is another important way[14].

\section{Experiments}

The experimental material was AZ31(Mg-3\%Al-1\%Zn0.5\%Mn) magnesium alloy sheet, which was produced by rolling process and then annealed at $400^{\circ} \mathrm{C}$ for $3 \mathrm{~h}$. Compressing deformation was finished by thermal simulator (Gleeble 3500). Compressing deformation temperature was $170^{\circ} \mathrm{C}$, and strain rate was $0.1 \mathrm{~mm} / \mathrm{min}$, and compression ratio was $11 \%$, $17 \%$ and $23 \%$, respectively.

\section{Analysis of Orientation Maps of Twined Grains}

The grain orientation maps of twined grains on the same area after compressed deformation (compression ratio was $0 \%, 11 \%, 17 \%$ and $23 \%$, respectively.) are shown as Fig. 1 . When the compression ratio was $23 \%$, the EBSD indexing rate was below $55 \%$.

In order to track and analyze the variation of grain orientation with the deformation, 15 grains are selected, which are numbered from 1 to 15 . The results show that almost all of the grains orientation change after the first compression (compression ratio being 11\%) and the majority of them appear completely twin, such as grains of 1, 2, 3, 4, 5 and 7. Only a few of them turn up partly twin, such as grains 12 and 15 .

(a)

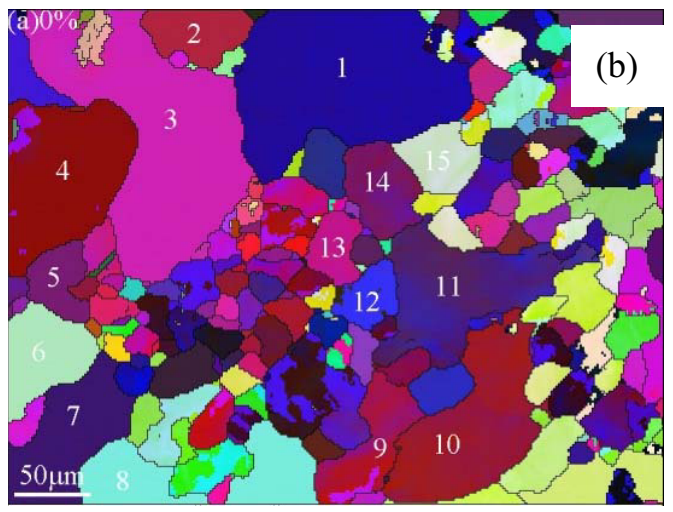




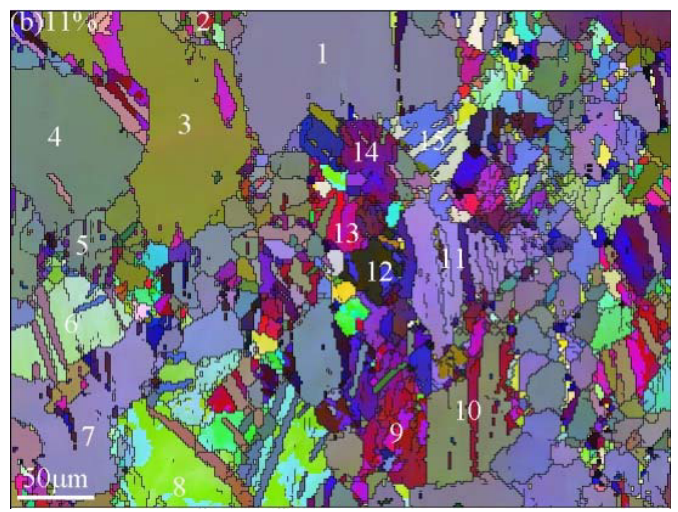

(c)
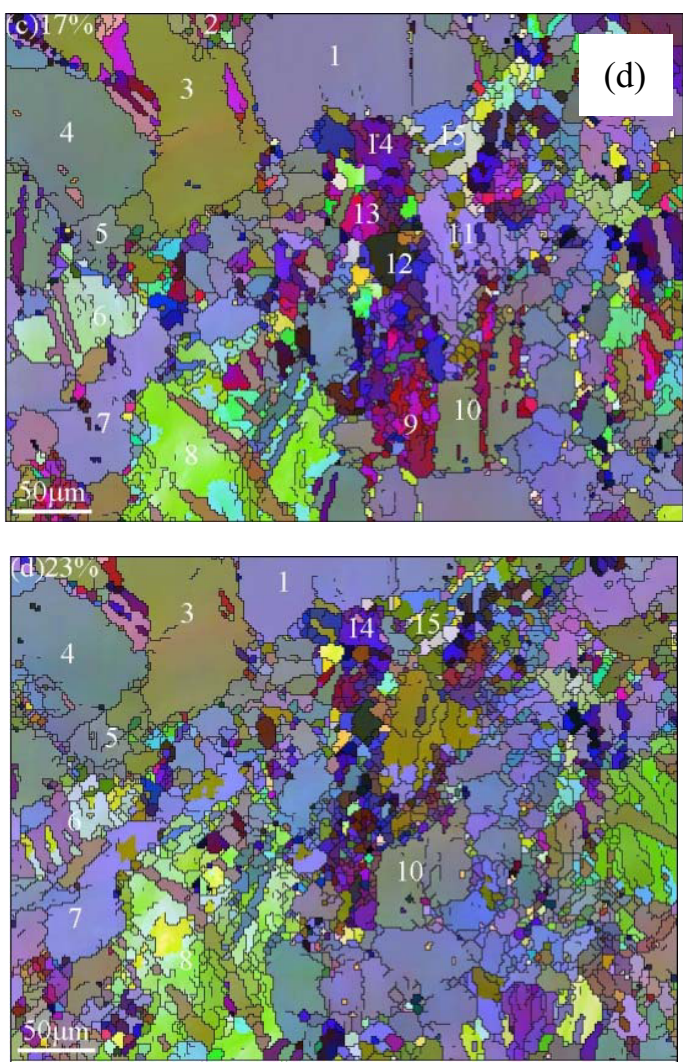

Fig.1 Grain orientation maps of twined grains by EBSD. (a, initial state; b, compression ratio 11\%; $\mathrm{c}$, compression ratio $17 \%$; $\mathrm{d}$, compression ratio $23 \%$.)

\section{Analysis of Pole and Inverse Pole}

In order to analyze the variation of texture of AZ31 magnesium alloy with different compression degree, the pole maps of AZ31 magnesium alloy are shown as Fig.2. Each group map includes $(0001)$ and $(10 \overline{10})$ pole figures. The typical rolling deformation texture before compression was shown as Fig.2a. Most $\mathrm{c}$ axis of grains is perpendicular 
with rolling direction. With the process of deformation, the basic texture begins to disappear. The reason was that most of grains appeared extension twins. The grain orientation turned $86.3^{\circ}$ around $<11 \overline{2} 0>$ axis. But there is no obvious variation when the compression ratio was from $11 \%$ to $23 \%$. The reason may be that the slip systems begin to start-up at high temperature when strain is large enough.

The inverse pole maps are shown as Fig.3. The compression axis is perpendicular with the $\mathrm{c}$ axis in ND direction (normal direction) before deformation. After three consecutive compressions, the compression axis is perpendicular with $\mathrm{c}$ axis in $\mathrm{RD}$ direction(rolling direction), and texture intensity increased. The reason was that most of grains appeared extension twins.

(a)
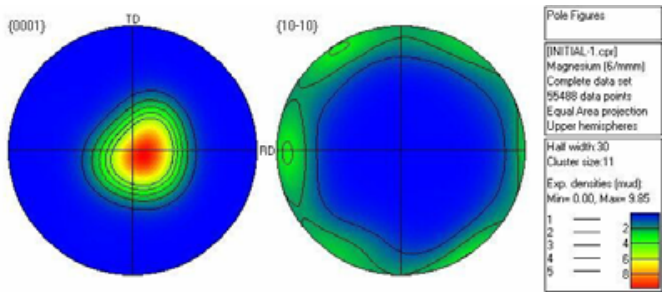

(b)
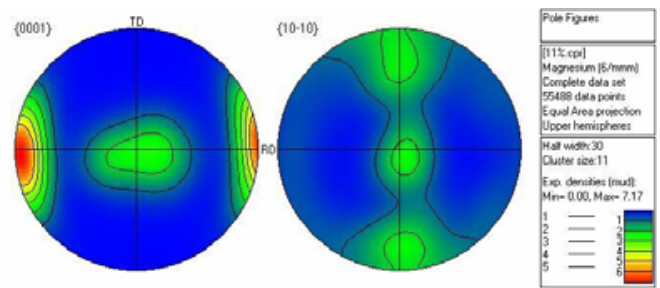

(c)
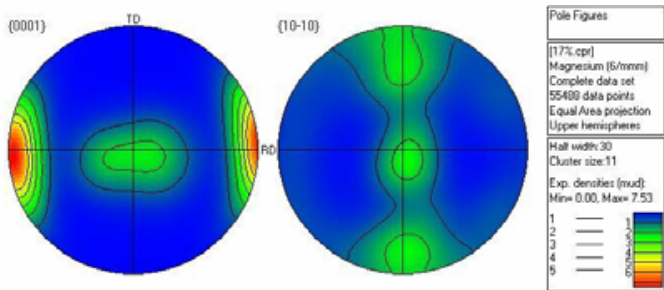

(d)

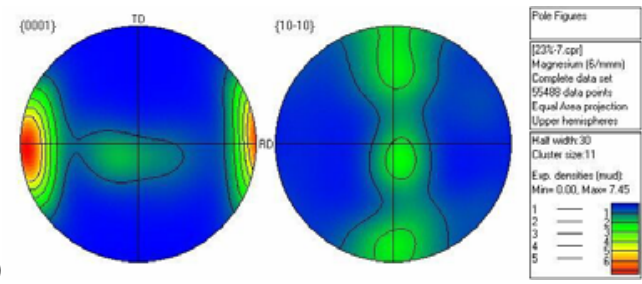

Fig.2 Pole maps ((a, initial state; b, compression ratio 11\%; c, compression ratio $17 \%$; d, compression ratio $23 \%$.) 
(a)
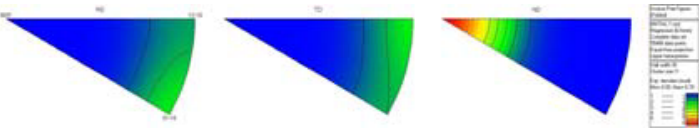

(b)
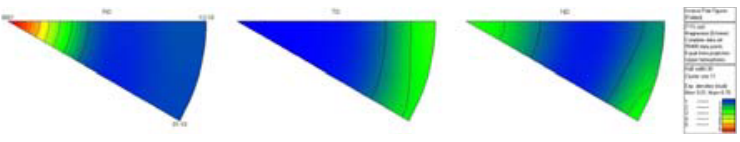

(c)
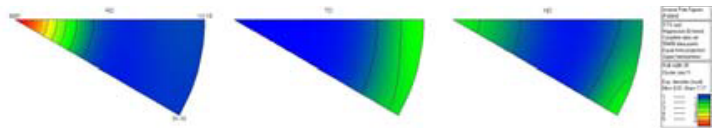

(d)
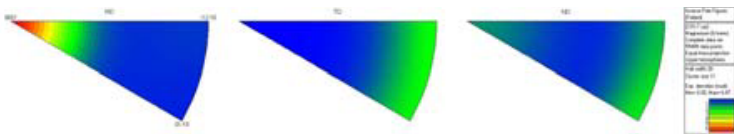

Fig.3 Inverse pole maps (a, initial state; b, compression ratio 11\%; c, compression ratio 17\%; d, compression ratio $23 \%$.)

\section{Misorientation Angle and Rotation Axis Distribution}

The distribution of orientation angle $\left(5^{\circ}-90^{\circ}\right)$ and rotation axis was shown as Fig.4. With the increasing of compression ratio, the frequency of misorientation angle significantly reduces at the range of $15^{\circ}-35^{\circ}$ and $50^{\circ}-60^{\circ}$. And when misorientation angle was at the range of $80^{\circ}-90^{\circ}$, the frequency of misorientation angle increased firstly and then reduces. When misorientation angle was at the range of $15^{\circ}-35^{\circ}$, the rotation axis distributes diffused between $<\overline{12} \overline{10}>$ and $<01 \overline{1} 0>$. When misorientation angle was at the range of $50^{\circ}-60^{\circ}$, the rotation axis mainly concentrated around $\left.<01 \overline{10}\right\rangle$, which was in agreement with the orientation $60^{\circ} /<10 \overline{10}>\left(\right.$ deviation of $\left.\pm 5^{\circ}\right)$ of the extension twin. When misorientation angle was at the range of $80^{\circ}-90^{\circ}$, the rotation axis mainly concentrates around $\langle\overline{1} 2 \overline{1} 0\rangle$, which was in agreement with the orientation $86.3^{\circ} /<\overline{12} \overline{1} 0>\left(\right.$ deviation of $\left.\pm 5^{\circ}\right)$ of extension twin. The results show that there are obvious extension twin during compression deformation.

(a)

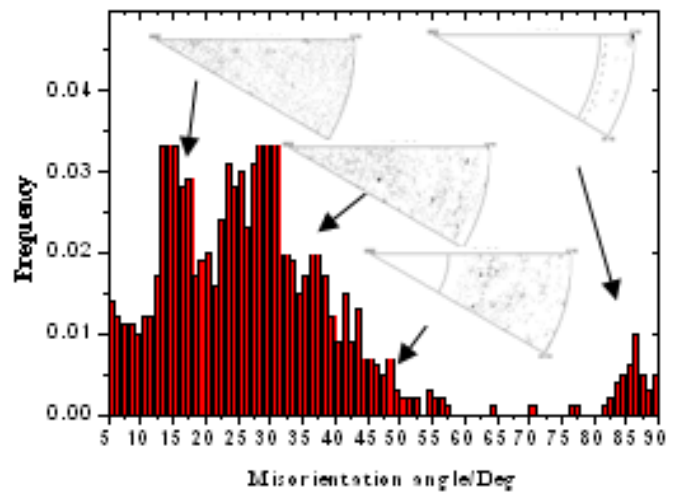

(b) 


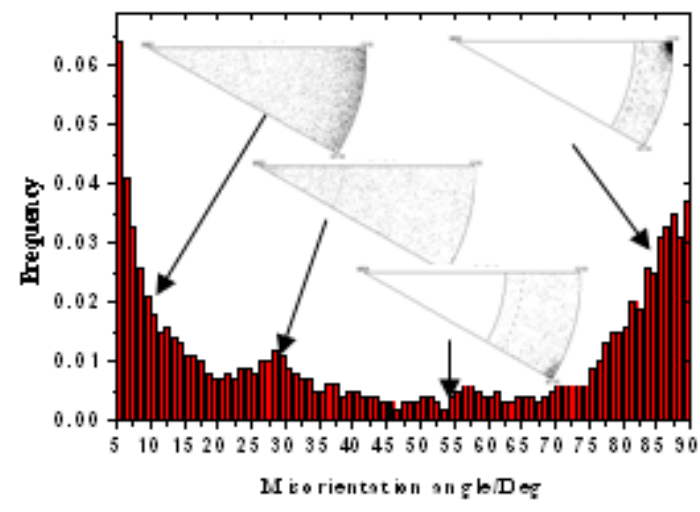

(c)

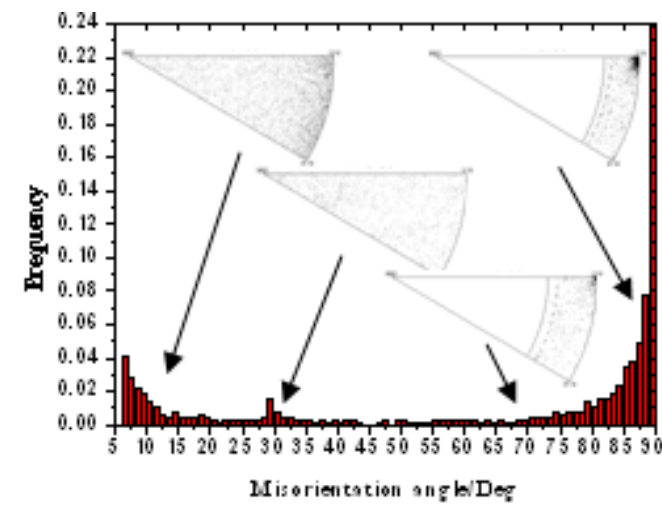

(d)

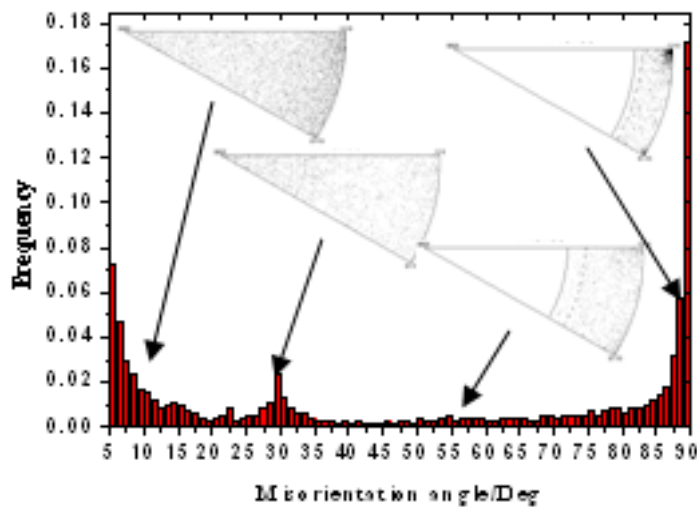

Fig.4 Misorientation angle and rotation axis distribution(a, initial state; b, compression ratio 11\%; compression ratio $17 \%$; $d$, compression ratio $23 \%$.)

\section{Conclusions}

(1) For AZ31 magnesium alloy sheet of rolled state, micro-texture was typical (0001) basic texture. When compression ratio was $11 \%$ and deformation temperature was $170^{\circ} \mathrm{C}$, the grain orientation changed significantly, and most of grains twined completely, a little grains twined partially, and the initial basal rolling texture weakened gradually.

(2) When misorientation angle was at the range of $50^{\circ}-60^{\circ}$, the rotation axis mainly 
concentrated around $\langle 01 \overline{10}\rangle$, which was in agreement with the orientation $60^{\circ} /<10 \overline{10}>\left(\right.$ deviation of $\left.\pm 5^{\circ}\right)$ of the extension twin. When misorientation angle was at the range of $80^{\circ}-90^{\circ}$, the rotation axis mainly concentrates around $<\overline{1} \overline{1} \overline{1} 0>$, which was in agreement with the orientation $86.3 \%<\overline{12} \overline{10} 0$ (deviation of $\pm 5^{\circ}$ ) of extension twin.

\section{Acknowledgements}

This project is supported by National Natural Science Foundation of China (Grant No. 51575366), and the Science and Technology Bureau of Shenyang city(F14-231-1-32).

\section{References}

1. Mordike B L, Ebert T. Magnesium Properties-Applications-Potential, Materials Science and Engineering. A302(2001)37-45.

2. Barnett M R, Keshavarz Z, Nave M. Microstructural features of rolled Mg-3Al-1Zn. Physical Metallurgy and Materials Science. 36(2005)1697-1704.

3. Keshavarz Z, Barnett M R. EBSD analysis of deformation modes in Mg-3Al-1Zn, Scripta Materialia. 55(2006)915-921.

4. Fu DingFa, Xu Fanyan, Xia Weijun. Effect of annealing of microstructure and mechanical properties of Magnesium AZ31, Natural Science Journal of Xiangtan University. 27(4)(2005)57-61.

5. Brown D W, Agnew S R, Bocrke M A. Internal strain and texture evolution during deformation twinning in magnesium, Materials Science and Engineering A. 399(1-2)(2005)1-12.

6. Han $\mathrm{J} \mathrm{H}$, Jee $\mathrm{K} \mathrm{K}$, Oh K H. Orientation rotation behavior during in situ tensile deformation of polycrystalline 1050 aluminum alloy, International Journal of Mechanical Sciences. 45(2003)1613-1618.

7. Watanabe H, Mukai T, Ishikawa K. Differential speed rolling of an AZ31 magnesium alloy and the resulting mechanical properties. Journal of Materials Science. 39(2004)1477-1485.

8. Chino Y, Mabuchi M, Kishihara R. Mechanical properties and press formability at room temperature of AZ31 Mg alloy processed by single roller drive rolling, Materials Transactions. 43(2002)2554-2561.

9. Yang Ping, Meng Li, Mao Weimin. Improvement of Formability of Magnesium Alloys by Pass Interval Annealing During Rolling, Transactions of Materials and Heat Treatment[J], 2005,(2):26-29.

10. Iwanaga $\mathrm{K}$, Tashiro $\mathrm{H}$, Okamoto $\mathrm{H}$. Improvement of formability from room temperature to warm temperature in AZ31 magnesium alloy, Journal of Materials Processing Technology. 155-156(2004)1313-1319.

11. Nave M D, Barnett M R. Microstructures and textures of pure magnesium deformed in plane-strain compression, Scripta Materialia. 51(2004)881-889.

12. TANG Wei-qin, ZHANG Shao-rui, FAN Xiao-hui. Texture and its effect on mechanical properties of AZ31 magnesium alloy, The Chinese Journal of Nonferrous Metals. 20(3)(2010)0371-0378.

13. JIN Xiao-kun, ZHENG Shen-bai, XU Yong, HANG Shi-hong. Effects of rolling process on microstructure of twin roll casting AZ31 magnesium alloy sheet, Transactions of Materials and Heat Treatment. 35(9)(2014)126-130.

14. DING Wen-jiang, JIN Li, WU Wen-xiang, DONG Jie. Texture and texture optimization of wrought $\mathrm{Mg}$ alloy, The Chinese Journal of Nonferrous Metals. 21(10)(2011)2371-2381. 